\title{
Structural characterization of the N-terminal domain of the Dictyostelium discoideum mitochondrial calcium uniporter
}

Yuan Yuan ${ }^{1 \dagger}$, Chan $\mathrm{Cao}^{2 \dagger}$, Maorong $\mathrm{Wen}^{1 \dagger}$, Min $\mathrm{Li}^{1}$, Ying Dong ${ }^{1}$, Lijie $\mathrm{Wu}^{3}$, Jian $\mathrm{Wu}^{4}$, Tanxing $\mathrm{Cui}^{2}$, Dianfan $\mathrm{Li}^{1 *}$, James J. Chou ${ }^{2 *}$, Bo OuYang ${ }^{1 *}$

${ }^{1}$ State Key Laboratory of Molecular Biology, CAS Center for Excellence in Molecular Cell Science, Shanghai Institute of Biochemistry and Cell Biology, Chinese Academy of Sciences; University of Chinese Academy of Sciences, 333 Haike Road, Shanghai 201203, P. R. China.

2 Department of Biological Chemistry and Molecular Pharmacology, Harvard Medical School, Boston, MA 02115, USA.

${ }^{3}$ Shanghai Institute for Advanced Immunochemical Studies and iHuman Institute, ShanghaiTech University, Shanghai 201210, China

${ }^{4}$ Ninth People's Hospital, Shanghai Jiao Tong University School of Medicine, Shanghai, 200125, China.

\section{Supporting Information (SI)}

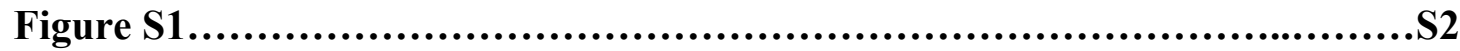

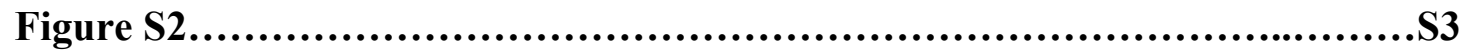

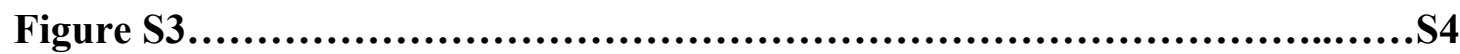

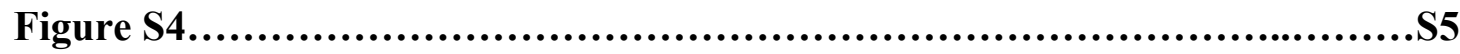

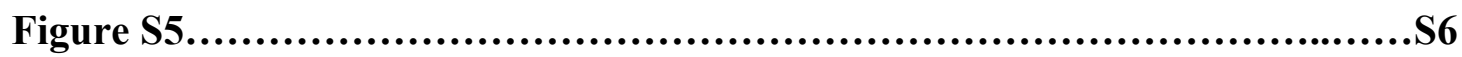

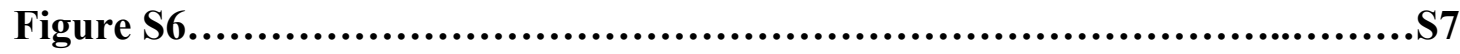

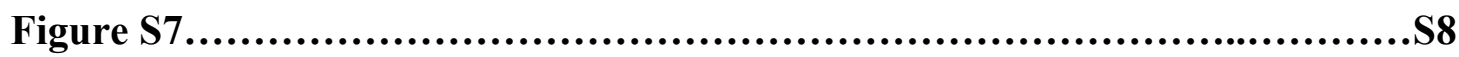

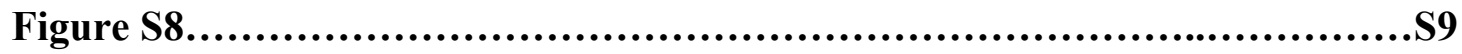

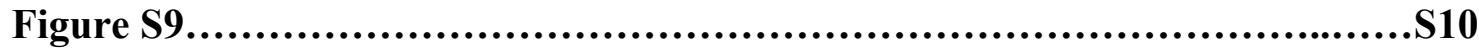

References............................................................................. 

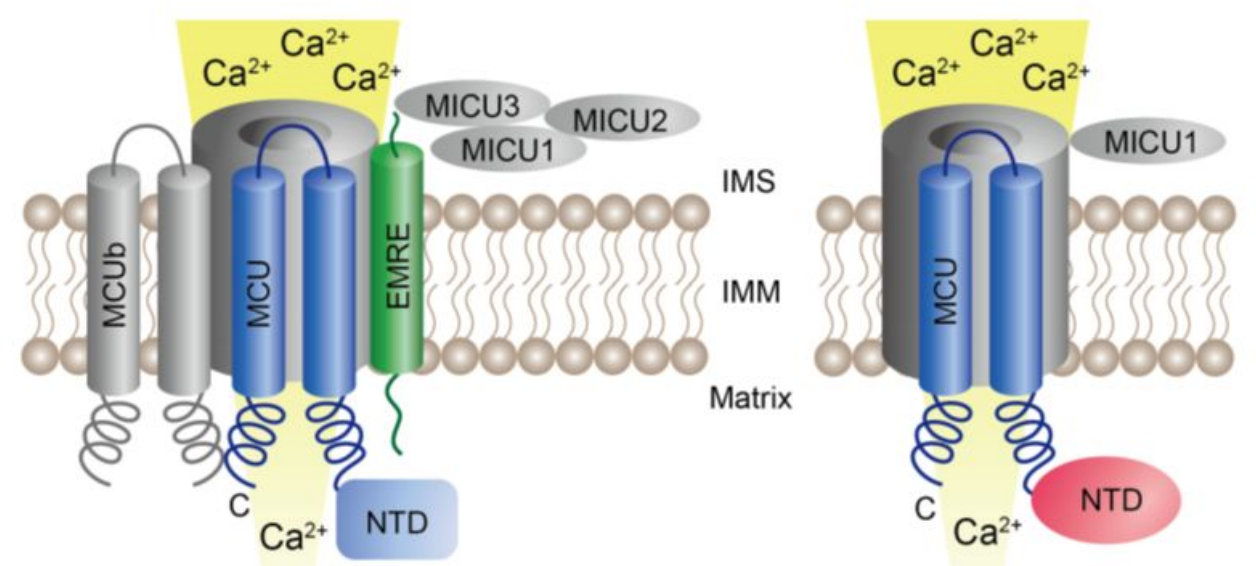

Figure S1. The components of the mitochondrial calcium uniporter complexes in Homo sapiens and Dictyostelium discoideum. The uniporter components in Homo sapiens consist of mitochondrial calcium uniporter protein (MCU), MCU regulatory subunit b (MCUb) and essential MCU regulator (EMRE), together with the intermembrane space (IMS) proteins mitochondrial calcium uptake protein 1 (MICU1), MICU2 and probably MICU3. The Dictyostelium discoideum uniplex proteins only contain MCU homologue and a putative MICU1 homologue. The major difference for the pore-forming component MCU in Homo sapiens and Dictyostelium discoideum lies in the N-terminal domain (NTD). 


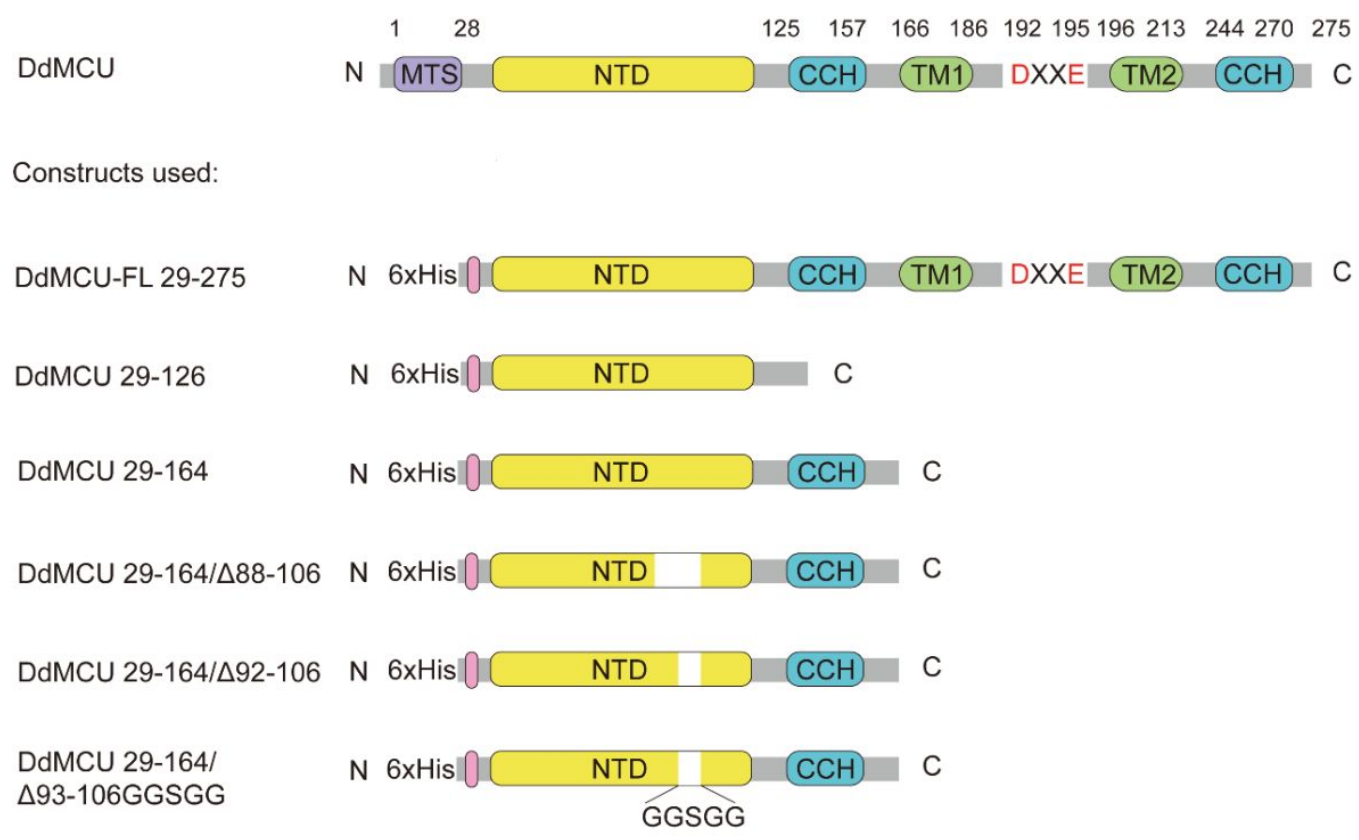

Figure S2. A linear overview of the domain organization for the original sequence of DdMCU and the constructs used in this paper. For each protein construct, different colors represent different domain: the predicted mitochondrial targeting signal (MTS) (purple), $6 \times$ His tag (pink), N-terminal domain (NTD) (yellow), transmembrane domain (TM) (green), the conserved DXXE motif, and coiled-coil helix ( $\mathrm{CCH})$ (blue). 
A

\begin{tabular}{|c|c|c|c|c|c|}
\hline \multicolumn{2}{|c|}{} & MaMCU-NTD & NFMCU-NTD & NcMCU-NTD & CyMCU-NTD \\
\hline HsMCU- & identity & $4.4 \%$ & $9.4 \%$ & $1.5 \%$ & $5.8 \%$ \\
\hline NTD & similarity & $8.1 \%$ & $13.6 \%$ & $2.4 \%$ & $9.5 \%$ \\
\hline $\begin{array}{c}\text { HsMCU- } \\
\text { NTD }\end{array}$ & Zscore & 10.2 & 10.2 & 10.8 & 10.9 \\
\hline & RMSD $(\AA)$ & 1.6 & 1.7 & 2.0 & 1.5 \\
\hline
\end{tabular}

C

-LLEEEEELLEEEEELLLLLLLLEEELLLLLLLHHHHHHHHLLLLLLEEELLLLLLLLLLLHHHLLL---LEEEEEL--LEEEEELLLLLLHHHHLLLLLLLLLI HsMCU-NTD _MVTVYYNGLPVISVRLPSRRERCQFTLKPISDSVGVLRQLQEEDRGIDRVAIYSPDGVRVAASTGIDLLLLD---DFKLVIN--DLTYHVRPPKRDLLSHENAATLVDVTL

CYMCU-NTD tTPSRLLKLVLPLSTVDH----APLALLVHPQ-QPLSYLERLIQAELP--EGEGKDEGEFVRTSPSTEIGDFIRDaarakEFEVEIEgSPGVIKVAVP-

1LLEEELLLLLLEEELL---_LEEEELLLL-LLHHHHHHHHHLL_-_LEEEEELLEELLLLLLLLLHHHhhhh1LEEEEL11LLEEEELLL

Figure S3. Structural alignment of MCU with different species homologues. Dali server was used to generate the structure alignment. (A) The sequence identity/similarity (top) and the z-score/RMSD values (bottom) of different fungal homologues comparing to HsMCU-NTD.

(B) Superimposing protein structures of HsMCU-NTD (blue, PDB code: 4XTB) with MaMCU-NTD (PDB code: 6C5R) (yellow), CyMCU-NTD (PDB code: 6DNF) (violet), NcMCU-NTD (PDB code: 6DT0) (green), NfMCU-NTD (PDB code: 6D7W) (orange) (C) The aligned secondary structure between HsMCU-NTD and CyMCU-NTD (H/h: helix, E/e: strand, L/l: coil). Uppercase means structurally equivalent positions with CyMCU-NTD. Lowercase means insertions relative to CyMCU-NTD. 
MGHVLG . GTLLAANRLAR ... PPAVVLGK . PRVCCWRASPWPVIVSSALOFSSSSARHTNYTSARYEARG MNCVRMRLQCRIMPSSNTLARWCLESPQKNLTLHR . . PRISNVALRQASTSVSPKTRETEAEAKAKKLDQKRLDEHE MRALVSRTPIAAALRSATLGSOCASIOYNSLNILDRLPQPLPSRSTRIRYTGVSTSGRVTQTCRRSTQLSASSRDKRE

DdMCU

MaMCU

NCMCU

CyMCU

NfMCU HsMCU

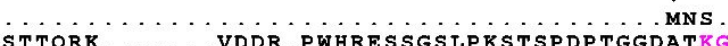

10 VI VDDR. PWHRESSGSLPKSTSPDPTGGDATKG

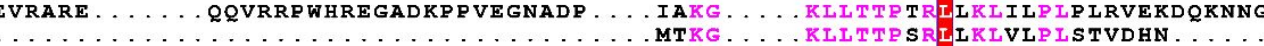
RLITTPTRLVRTENTRLTTS . . . . PQSAEPDPLERLEVKKVQQQHENEKDDSGRDTKSGGKVAKAMTKGDTIAGKLITTPSRLFKLIIPITTIN . . . . . .

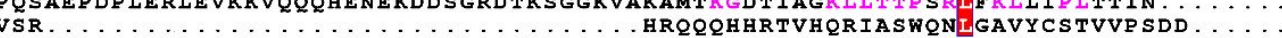

$$
30 \text { elelecelecelecelee }
$$

$$
\stackrel{\beta 1}{\rightarrow} \stackrel{\alpha 2}{2}{ }_{60}
$$

e. . . . . .

DdMCU

$\mathrm{NCMCU}$

CyMCU

HsMCU

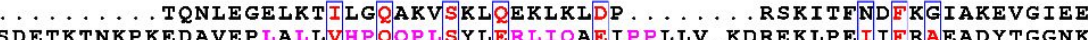

RNNEYGRS I SLNSDIOPLALI I HPOLPI SYYVERI I A L LP PVVE . NGOEKIPNVYFRAEDSEOGDQKP TSRAEARSKD

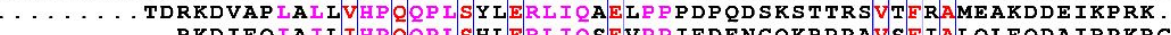

......RKDIEQIAILIHPQQPLSHLERLIQSEVPPIEDENGQKRP PAVSFIALQLEQDAIRPKRGM.YEGTD VTVVYQNGLPVISVRIPSRRERCQFTLKPISDSVGVFLRQLQEEDRGIDRVAIYSPDGVRV . .
$\overrightarrow{\beta 1}$
$\beta 2$
$\beta 3$
elevelele
B4

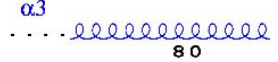

$\stackrel{\alpha 4}{100} \stackrel{\beta 2}{\longrightarrow}$

$\alpha 5$

$\ldots$. KEINSVSNALAQ $\ldots . . . .$. SGSI IYLPNSINENIKTSVFTKPAHI . . . . YQSLEHILDIENKGVG

\section{0}

110 DGGRRESTH DGGEPSEYNNLSHVAS............

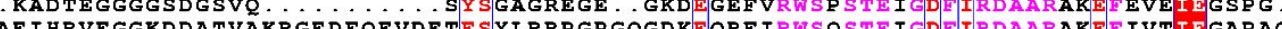
作 .....AASTGIDLLLLD ........ DEKLVINDLTYHVRPEKRDLLSHENAATLNDVKTLVQQLYTTLCIEQHQLN

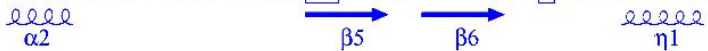

130 140 150 160 170 180 190

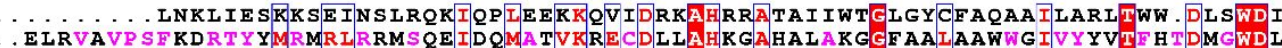
ELRVAVP S KDRTYYMRMRI RRMSQEIDQMA TVKRECDILAHKGAHALAKGGFAAI AAWWG IVYYVTFHTDMGWDI EMRVSVP SF GDRTYYMRQRI RKMS SEIDGIAKIKHECDILAHRSAHRLAKGGFGLIAGWWGVVYYVTFHTEFGWDI

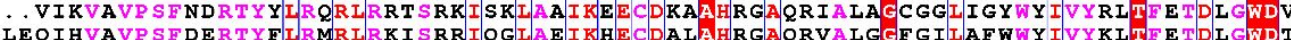
NAMCU LEQIHVAVPSFDERTYFIRMRLRKISRRI QGIAEIKHECDALAHRGAQRVALGGFGIIAFWWYIVYKLTFETIIGWDT
HSMCU

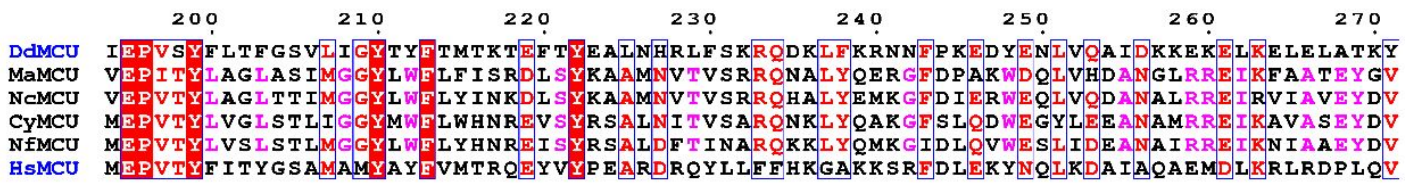

DdMCU DHTH

a

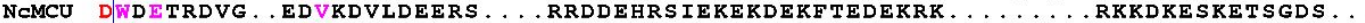

CYMCU DWNETQDEGGDEKVTKALRDERKNNNGTKNKSKEGEEDDEDD

NFMCU DWDERKDEQ.DDRVTEALKKERRLKNGSOKEERPKDDRDDDQY QY . VR. VR. QYQYQYQYQYVGVGVGVRVRYYVG HSMCU HLPLRQIGEKD.

Figure S4. Sequence alignment of different MCU homologues. The comparison of the sequence HsMCU with the fungal homologues and the comparison of the secondary structures of DdMCU-NTD and HsMCU-NTD using ClustalW and ESPript ${ }^{l}$ programs. The secondary structure elements on the top correspond to the DdMCU-NTD and the secondary structure elements below correspond to HsMCU-NTD. 
A

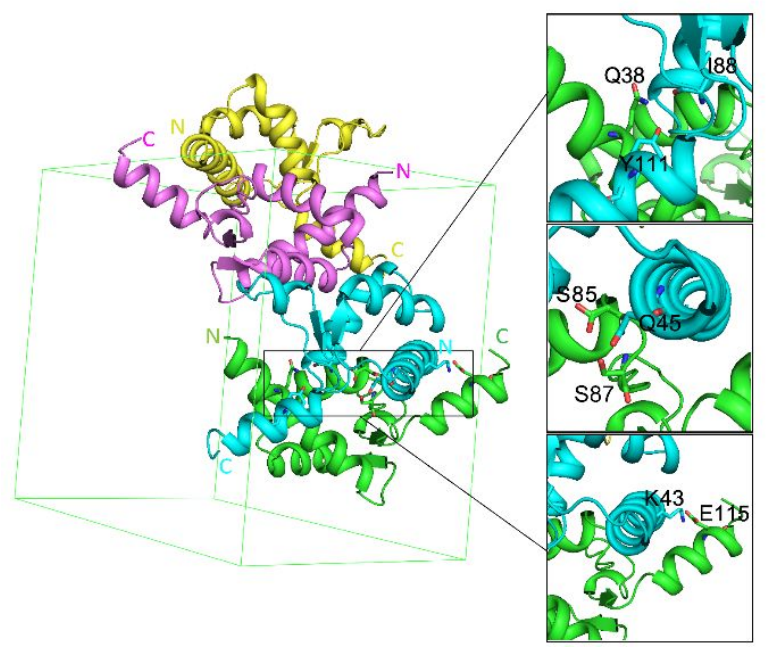

B

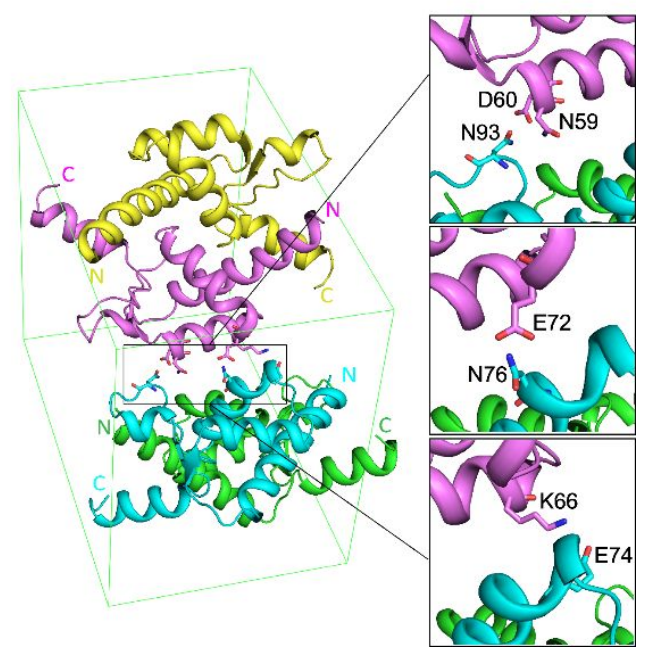

Figure S5. The packing interfaces of DdMCU-NTD in the unit cell. Two interfaces are identified, including interface I (A) containing three pairs of inter-molecular hydrogen bonds formed by Q38-I88/Y111, K43-E115 and Q45-S85/S87 and interface II (B) containing three pairs of inter-molecular interactions formed by N59/D60-N93, K66-E74, E72-N76. Detailed views of the pairs of inter-molecular interactions are shown on the right side. 

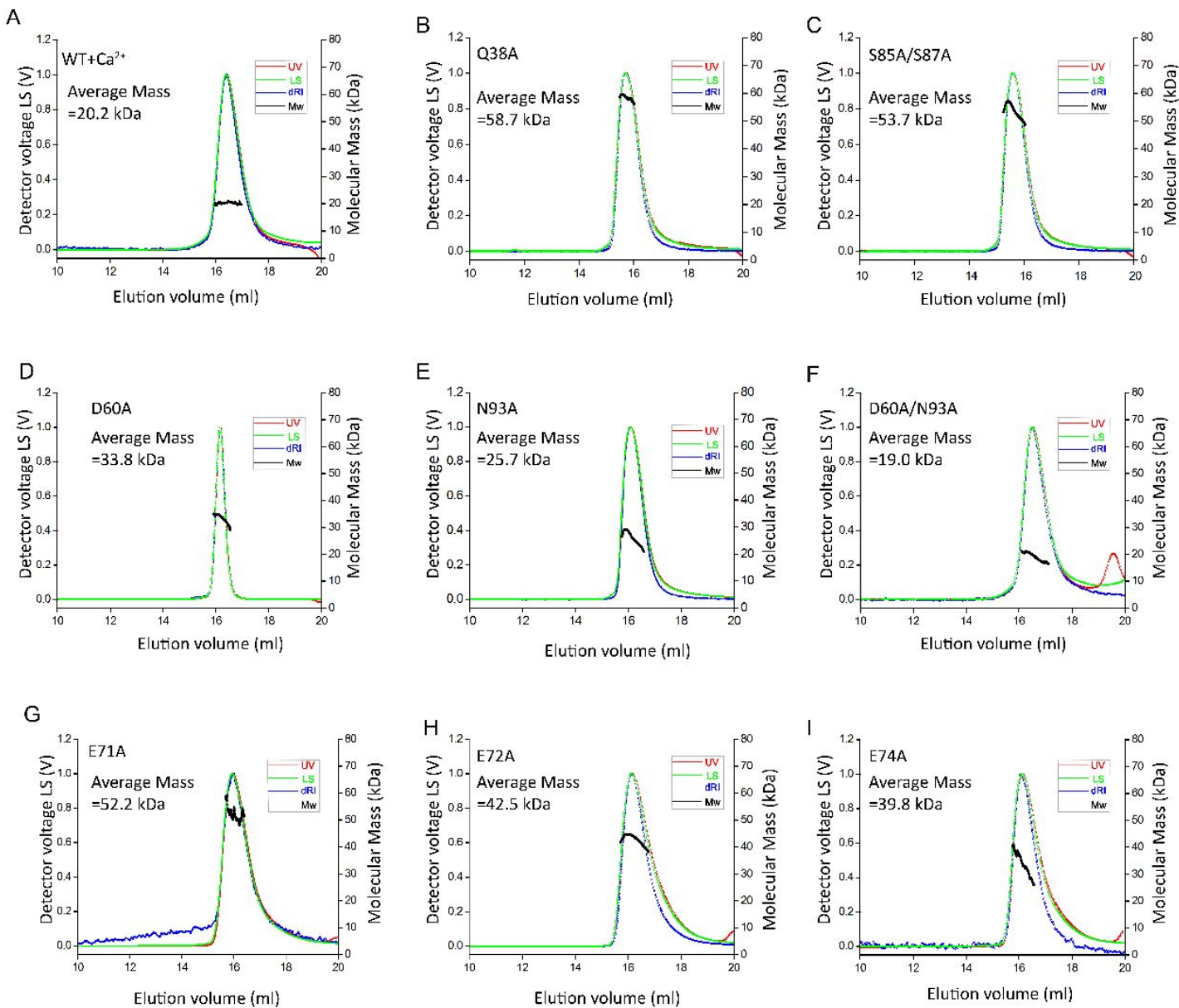

Figure S6. SEC-MALS analysis of different mutants. Chromatograms show the readings from the light scattering at $90^{\circ}$ (green), refractive index (blue), and UV (red) detectors. The left and right axes represent the light scattering detector reading and molecular weight, respectively. The black curve represents the calculated molecular weight that calcium addition to WT shifts the average molecular weight to $20.2 \mathrm{kDa}(\mathrm{A})$ and the average weight of the elution peaks of mutants Q38A (B), S85A/S87A (C), D60A (D), N93A (E), D60A/N93A (F), E71A (G), E72A (H), and E74A (I) are 58.7 kDa, 53.7 kDa, $33.8 \mathrm{kDa}, 25.7 \mathrm{kDa}, 19.0$ $\mathrm{kDa}, 52.2 \mathrm{kDa}, 42.5 \mathrm{kDa}$ and $39.8 \mathrm{kDa}$, respectively. 

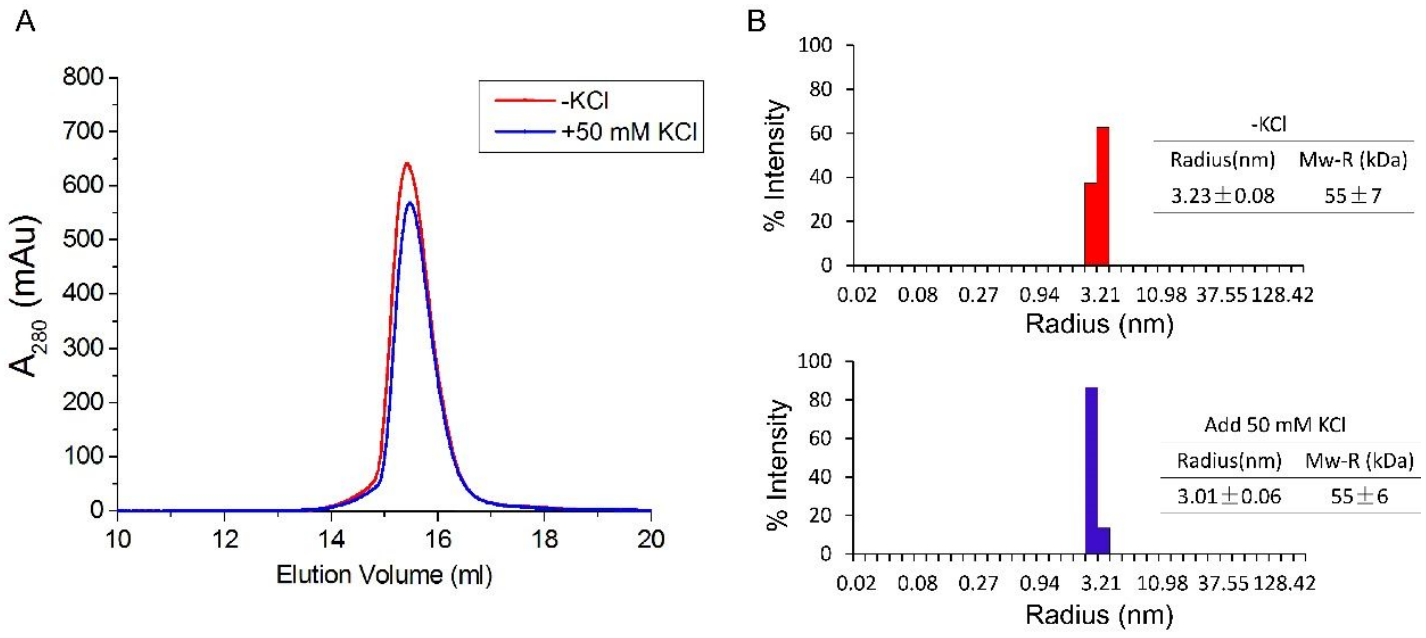

Figure S7. Potassium effects on DdMCU-NTD oligomerization. (A) Gel-filtration analysis of the DdMCU-NTD in the absence (red) and presence (blue) of $50 \mathrm{mM} \mathrm{K}^{+}$(Superdex 200 10/300 GL column (GE Healthcare)). (B) DLS intensity particle size distribution of DdMCU-NTD in the absence (red) or presence (blue) of $50 \mathrm{mM} \mathrm{K}^{+}$. 

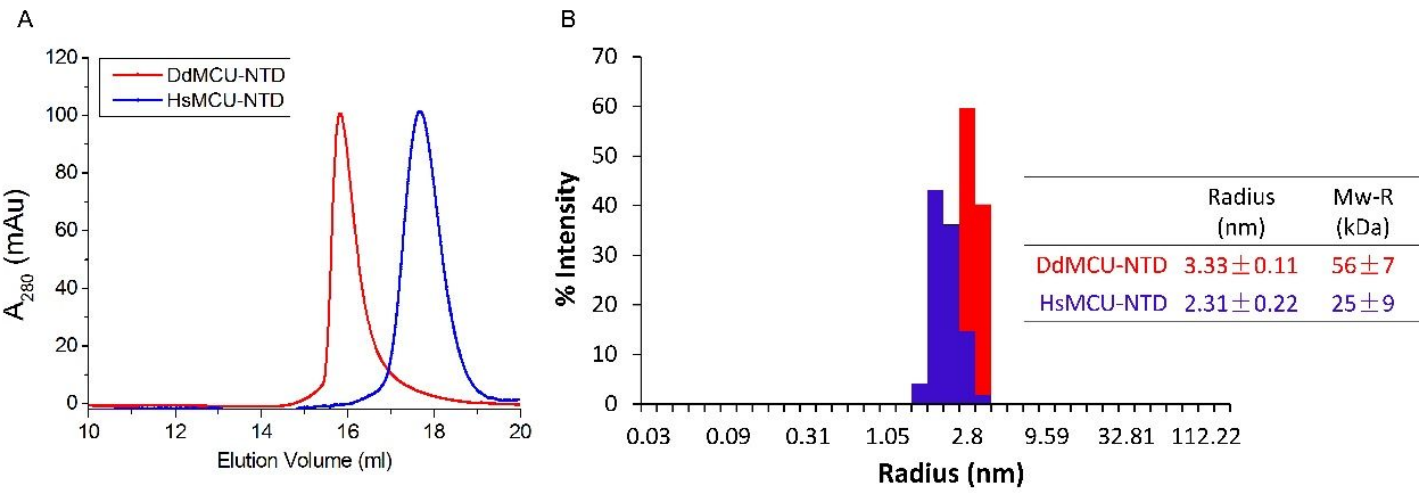

Figure S8. The oligomerization of DdMCU-NTD and HsMCU-NTD. (A) Gel-filtration results of DdMCU-NTD and HsMCU-NTD. (B) DLS results of DdMCU-NTD and HsMCU-NTD. 

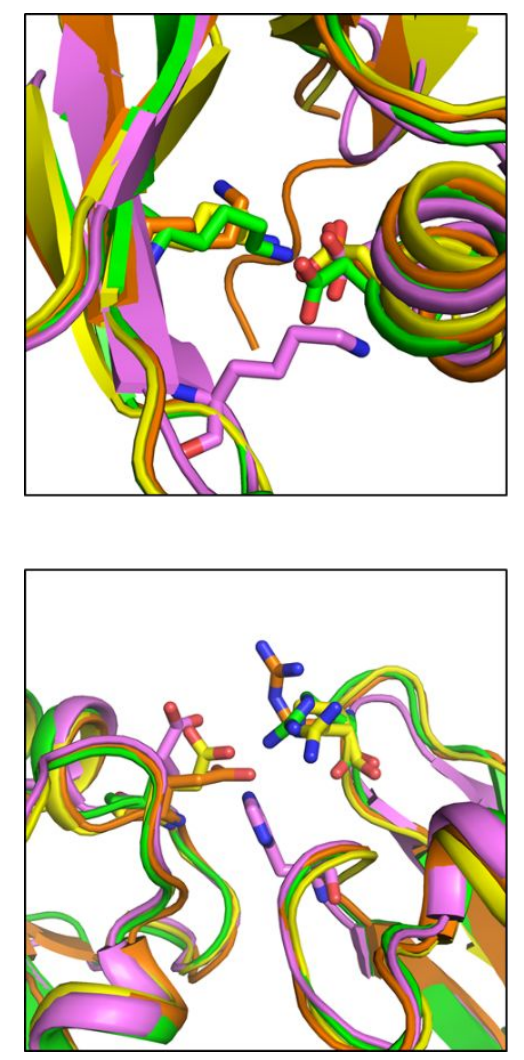

MaMCU R110-E235

CyMCU H40-E127

NcMCU R117-E257

NfMCU R136-E268

Figure S9. The conserved atomic interaction in the interface of two NTD subunits of the four fungal homologues. The salt bridge formed between an Asp and a Lys is identified in MaMCU-NTD (yellow), CyMCU-NTD (violet), NcMCU-NTD (green), NfMCU-NTD (orange). 


\section{References:}

1. Robert, X., and Gouet, P. Deciphering key features in protein structures with the new ENDscript server, Nucleic Acids Res. 2014, 42, W320-324. 